\title{
FRACTALISATION DU BASSIN D'ATTRACTION DANS L'ALGORITHME DE NEWTON MODIFIÉ
}

\author{
Mohamed LAmine SAHARI ${ }^{1}$ AND IlHem DJELlit ${ }^{1}$
}

\begin{abstract}
Newton's method is a root-finding algorithm and Newton basins is the set of initial guesses that lead to one root of polynomial on the complex plane. A boundary of Newton basins are fractals and called Julia set. In this work we introduce a modification on the algorithm and we show that the fractal aspect of basins is preserved.

Résumé. L'algorithme de Newton appliqué à la résolution d'équations algébriques à variable complexe génère une suite dotée d'un comportement chaotique qui se traduit par la "fractalisation" de la frontière du bassin d'attraction des solutions. Dans ce travail on introduit une modification sur l'algorithme et on montre que l'aspect fractal des bassins est preservé.
\end{abstract}

\section{INTRODUCTION}

L'étude des bassins d'attraction d'une récurrence générée par l'algorithme de Newton appliqué à la résolution des équations quadratiques à variable complexe a été considérée initialement par Schröeder (1871). Le cas cubique

$$
a z^{3}+b z^{2}+c z+d=0 ;(a, b, c, d) \in \mathbb{C}^{4}
$$

a été développé par Cayley (1879). Les travaux de Cayley [4] ont été repris ensuite par le mathématicien français Gaston Julia [6], pour fonder une théorie sur les ensembles qui portent son nom. Dans ce travail on examine le comportement chaotique d'une récurrence générée par l'algorithme de Newton sur une équation cubique de la forme

$$
z^{3}-1=0
$$

On cherche à étudier l'influence d'une modification dans l'algorithme de Newton sur le bassin d'attraction des solutions de (1).

\section{Notations ET DÉFinitions}

On note $\mathbb{C}$ le plan complexe, la sphère de Riemann $\overline{\mathbb{C}}$ est définie comme union de $\mathbb{C}$ et le point à l'infini $\infty$ $(\overline{\mathbb{C}}=\mathbb{C} \cup\{\infty\})$.

Soit une récurrence complexe définie par

$$
z_{k+1}=g\left(z_{k}, \beta\right) \equiv g\left(z_{k}\right)
$$

Où $g: \overline{\mathbb{C}} \rightarrow \overline{\mathbb{C}}$ est une fonction holomorphe non constante et $\beta$ un paramètre dans $\mathbb{C}$.

\footnotetext{
1 Département de Mathématiques, Université Badji-Mokhtar. B.P. 12, Annaba 23.000. Algérie;

e-mail: mlsahari@yahoo.fr \& e-mail: i_djellit@hotmail.com
} 
Définition 2.1. Un point $\alpha \in \overline{\mathbb{C}}$ est dit point fixe pour $g$ si

$$
g(\alpha)=\alpha,
$$

il est dit attractif si

$$
\left|\frac{d}{d z} g(z)\right|_{z=\alpha}<1
$$

répulsif si

$$
\left|\frac{d}{d z} g(z)\right|_{z=\alpha}>1 .
$$

Définition 2.2. Le bassin d'attraction $\mathcal{A}(\omega)$ d'un point fixe attractif $\omega$, associé à une fonction $g$ est défini par

$$
\mathcal{A}(\omega)=\left\{z \in C: g^{k}(z) \underset{k \rightarrow \infty}{\rightarrow} \omega\right\}
$$

Définition 2.3. On définit l'ensemble de Julia remplie, noté $K(g)$ par

$$
K(g)=\left\{z: g^{k}(z) \nrightarrow \infty, k \rightarrow \infty\right\}
$$

Définition 2.4. L'ensemble de Julia de g est la frontière de $K(g)$, notée $\mathcal{J}(g)$. L'ensemble de Fatou $\mathcal{F}(g)$ est définit comme le complémentaire dans $\overline{\mathbb{C}}$ de $\mathcal{J}(g)$.

\section{L'Algorithme de NeWton}

On veut résoudre l'équation polynomiale à coefficients réels ou complexes à valeurs dans $\mathbb{C}$

$$
f(z)=0 .
$$

On construit alors une suite récurrente $\left\{z_{k}\right\}_{k=0,1,2, \ldots}$ de telle façon que $f\left(z_{k}\right) \rightarrow 0$. Supposons connue l'itérée courante $z_{k}$; l'équation (2) linéarisée en $z_{k}$ donne

$$
f(z)=f\left(z_{k}\right)+\left(z-z_{k}\right) f^{\prime}\left(z_{k}\right)
$$

la solution de cette équation est l'itérée suivante $z_{k+1}$, donne par

$$
z_{k+1}=z_{k}-\frac{f\left(z_{k}\right)}{f^{\prime}\left(z_{k}\right)}
$$

Si

$$
f(z)=z^{n}-1, n \in \mathbb{N} .
$$

En remplaant dans (3), on obtient l'équation dite de Newton

$$
(n-1) z_{k}^{n}-n z_{k+1} z_{k}^{n-1}+1=0
$$

d'où l'algorithme de Newton

$$
z_{k+1}=z_{k}+\frac{1-z_{k}^{n}}{n z_{k}^{n-1}} .
$$


Théorème 3.1. Supposons que $f$ est deux fois continûment dérivable et appelons $z^{*}$ un zéro de $f$. Alors si $f^{\prime}\left(z^{*}\right) \neq 0$ et $z_{0}$ est suffisamment proche de $z^{*}$. La suite $\left\{z_{k}\right\}_{k=0,1, \ldots}$ engendrée par l'algorithme de Newton converge vers $z^{*}$ et la vitesse de convergence est quadratique, i.e.

$$
\lim \frac{\left\|z_{k+1}-z^{*}\right\|}{\left\|z_{k}-z^{*}\right\|^{2}}=M<+\infty
$$

où $M$ est une constante positive.

\section{Application sur le cas cubique "Problème de Cayley"}

C'est le cas $n=3$; donc

$$
f(z)=z^{3}-1 .
$$

Il est facile de calculer les trois racines du polynôme précédent

$$
\xi_{j}=e^{\frac{2}{3}(j-1) \pi i}, j=1,2,3
$$

ou

$$
\xi_{1}=1, \xi_{2,3}=-\frac{1}{2} \pm i \frac{1}{2} \sqrt{3} .
$$

L'équation de Newton s'écrit alors sous la forme

$$
2 z_{k}^{3}-3 z_{k+1} z_{k}^{2}+1=0,
$$

plus explicitement

$$
z_{k+1}=\mathcal{N}\left(z_{k}\right)=z_{k}+\frac{1-z_{k}^{3}}{3 z_{k}^{2}} .
$$

On note $z_{+}=x_{+}+i y_{+}$et $z=x+i y$ au lieu de $z_{k+1}=x_{k+1}+i y_{k+1}$ et $z_{k}=x_{k}+i y_{k}$. Si on remplace dans (4), en séparant la partie réelle de la partie imaginaire et en effectuant un calcul mécanique, on trouve

$$
\left\{\begin{array}{l}
x_{+}=\frac{2 x^{5}+4 x^{3} y^{2}+2 x y^{4}+x^{2}-y^{2}}{3\left(x^{2}+y^{2}\right)^{2}} \\
y_{+}=\frac{2 y\left[\left(x^{2}+y^{2}\right)^{2}-x\right]}{3\left(x^{2}+y^{2}\right)^{2}}
\end{array}\right.
$$

Il est facile de vérifier que les solutions $\xi_{1}, \xi_{2}$ et $\xi_{3}$ sont les seuls points fixes attractifs. Dans $[2,3,9]$ on montre que la frontière des bassins d'attraction $\partial \mathcal{A}\left(\xi_{j}\right), j=1,2,3$ est d'un aspect fractal de plus $\mathcal{J}(\mathcal{N})=\partial \mathcal{A}\left(\xi_{1}\right)=$ $\partial \mathcal{A}\left(\xi_{2}\right)=\partial \mathcal{A}\left(\xi_{3}\right)($ FIG. 1).

\section{Modification de L'Algorithme de Newton et Globalisation}

On note que l'algorithme (4)-(5) converge seulement pour un $z$ proche de la solution (Théorème 3.1), au delà on n'a pas de convergence globale. Pour cela, on peut interpréter la quantité $\frac{1-z^{3}}{3 z^{2}}$ comme une direction, le long de laquelle on effectue un déplacement $\lambda>0$ pour forcer la convergence globale. Un nouveau algorithme est donc proposé

$$
z_{+}=\mathcal{N}(z, \lambda)=z+\lambda d
$$

avec $d=\frac{1-z^{3}}{3 z^{2}}$. 

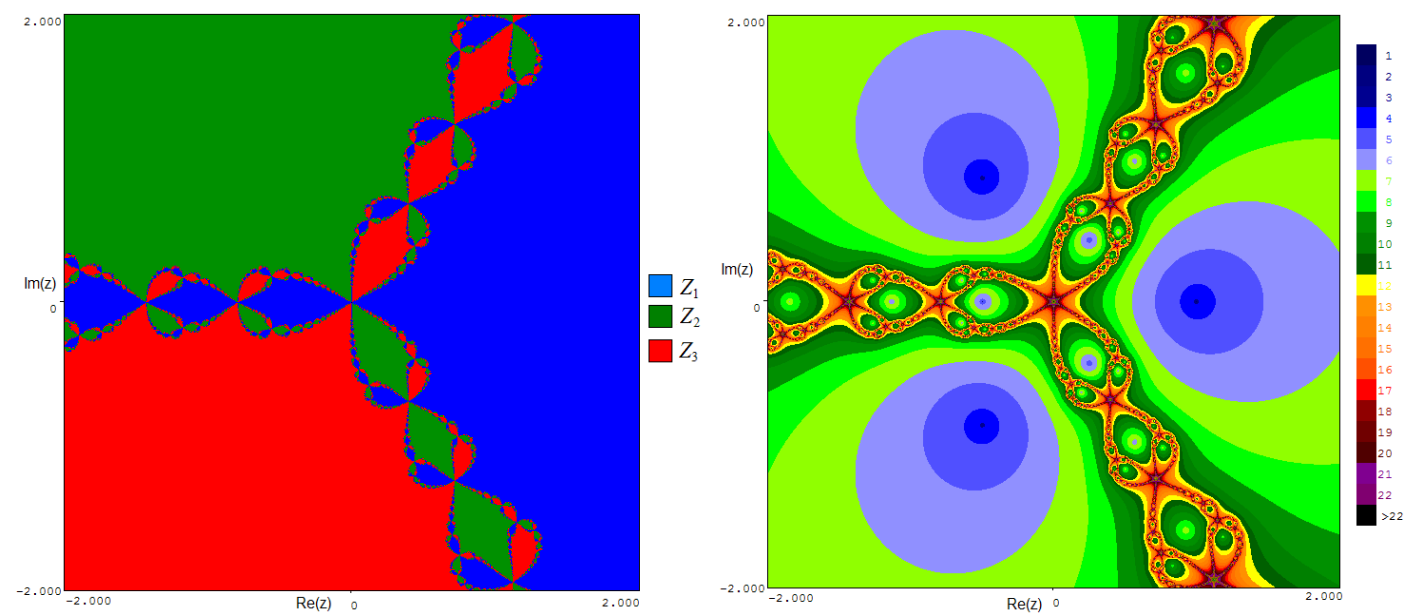

Figure 1. Les bassins d'attraction des trois solutions de l'équation $z^{3}-1=0$.

La projection de la quantité (6) sur les axes réel et imaginaire donne la récurrence suivante dans $\mathbb{R}^{2}$

$$
\left\{\begin{array}{l}
x_{+}=\frac{(3-\lambda)\left(x^{5}+2 x^{3} y^{2}+x y^{4}\right)+\lambda\left(x^{2}-y^{2}\right)}{3\left(x^{2}+y^{2}\right)^{2}} \\
y_{+}=\frac{y\left[(3-\lambda)\left(x^{2}+y^{2}\right)^{2}-2 \lambda x\right]}{3\left(x^{2}+y^{2}\right)^{2}}
\end{array}\right.
$$

En traçant les bassins d'attraction des solutions de l'équation (1) suivant l'algorithme modifié (6)-(7) avec des valeurs différentes de $\lambda$, on s'aperçoit qu'il existe des points où la convergence est obtenue après peu d'itérations (FIG. 2). Les figures (FIG. 3 (a)-(b)) montrent l'influence du choix de $\lambda$ sur $k_{\max }$ (le nombre d'itérations avant la convergence) avec des points initiaux qui appartiennent à l'ensemble de Julia $\mathcal{J}(\mathcal{N})$, considéré comme l'union de points non-convergeants. Dans la littérature $[1,8], \lambda$ est pris généralement comme solution approchée du problème de minimisation suivant

$$
\min _{\lambda>0}|f(z+\lambda d)|^{2}
$$

C'est ce qu'on appelle, recherche linéaire (R.L.) et plusieurs formules sont proposées pour la mise en œuvre de (15); parmi elles:

1) R.L. d'Armijo: Si on pose $\theta(\lambda) \equiv|f(z+\lambda d)|^{2}$ et $\theta^{\prime}(\lambda) \equiv \frac{d}{d \lambda} \theta(\lambda)$, le pas choisi $\lambda_{A}$ doit vérifier l'inégalité

$$
\theta\left(\lambda_{A}\right) \leq m \theta^{\prime}(0) \lambda_{A}+\theta(0)
$$

2) R.L. de Wolfe : Le pas choisi $\lambda_{W}$ doit vérifier les deux inégalités suivantes

$$
\left\{\begin{array}{l}
\theta\left(\lambda_{W}\right) \leq m_{1} \theta^{\prime}(0) \lambda_{W}+\theta(0) \\
\theta^{\prime}\left(\lambda_{W}\right) \geq m_{2} \theta^{\prime}(0)
\end{array}\right.
$$

avec $0<m_{1}<m_{2}<1$ et $0<m<1$.

Les tests numériques (FIG. 4) montrent que de tels procédés améliorent les performances de l'algorithme de Newton tout en préservant l'aspect fractal du bassin d'attraction des solutions. Si $\lambda$ est pris dans $\mathbb{C}$, la recurrence 

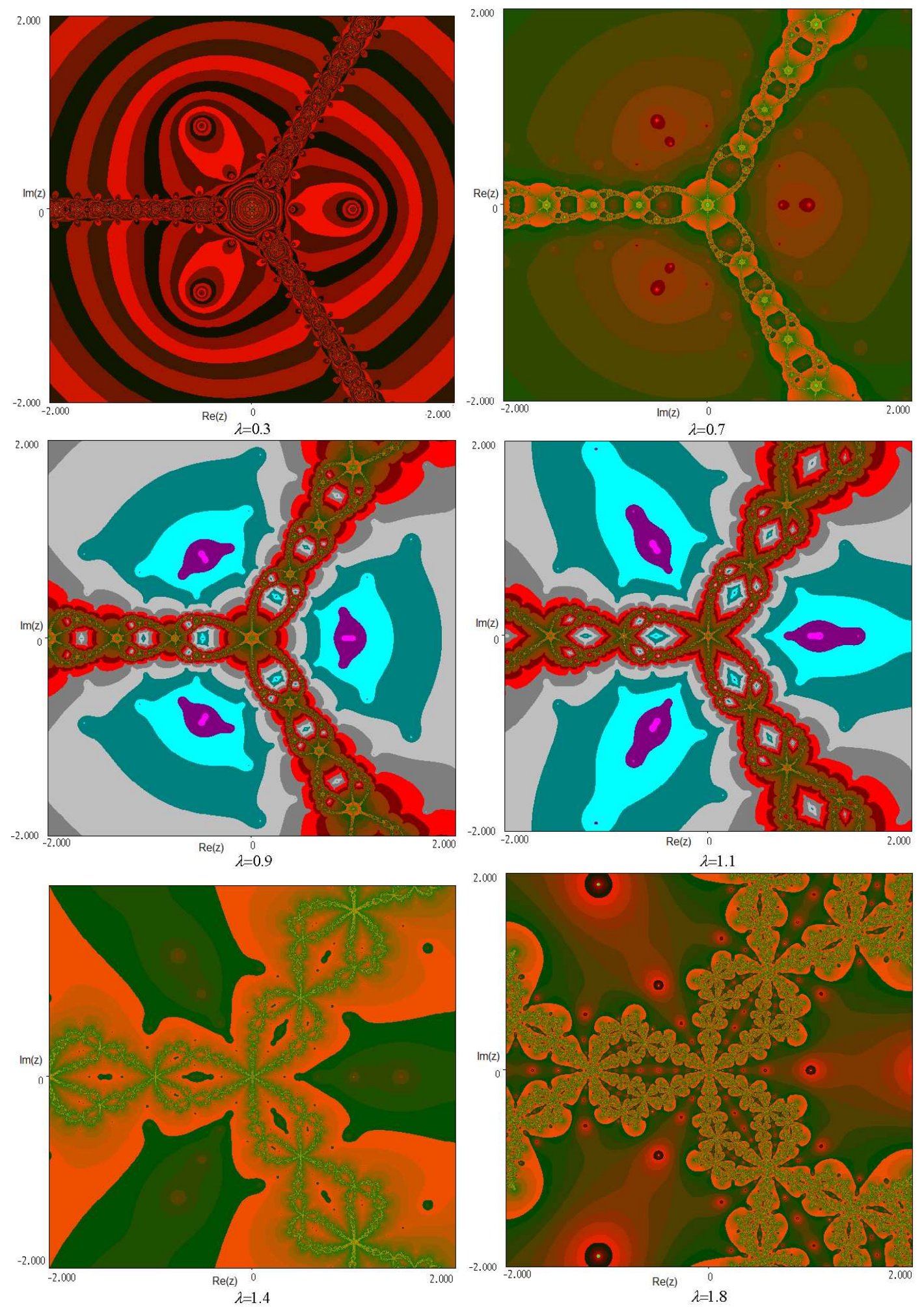

Figure 2. Apparition de structures fractales, variation du paramètre positif $\lambda$. 

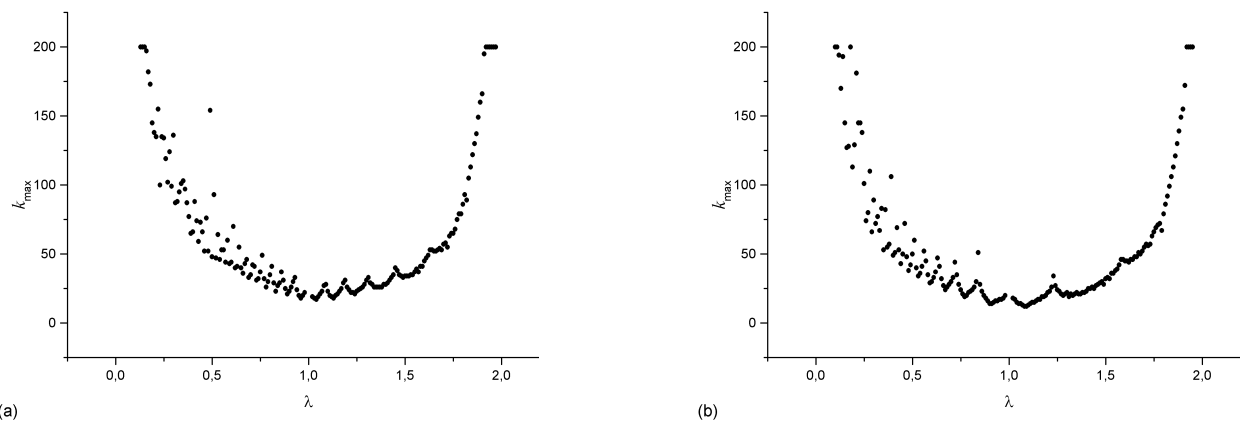

FiguRE $3 . k_{\max }(\lambda)$ pour l'algorithme de Newton modifié initié en : (a) $z_{0}=29.3443865317+$ $i \cdot 50.82596840$. (b) $z_{0}=2.572222299-i \cdot 4.455219707$.
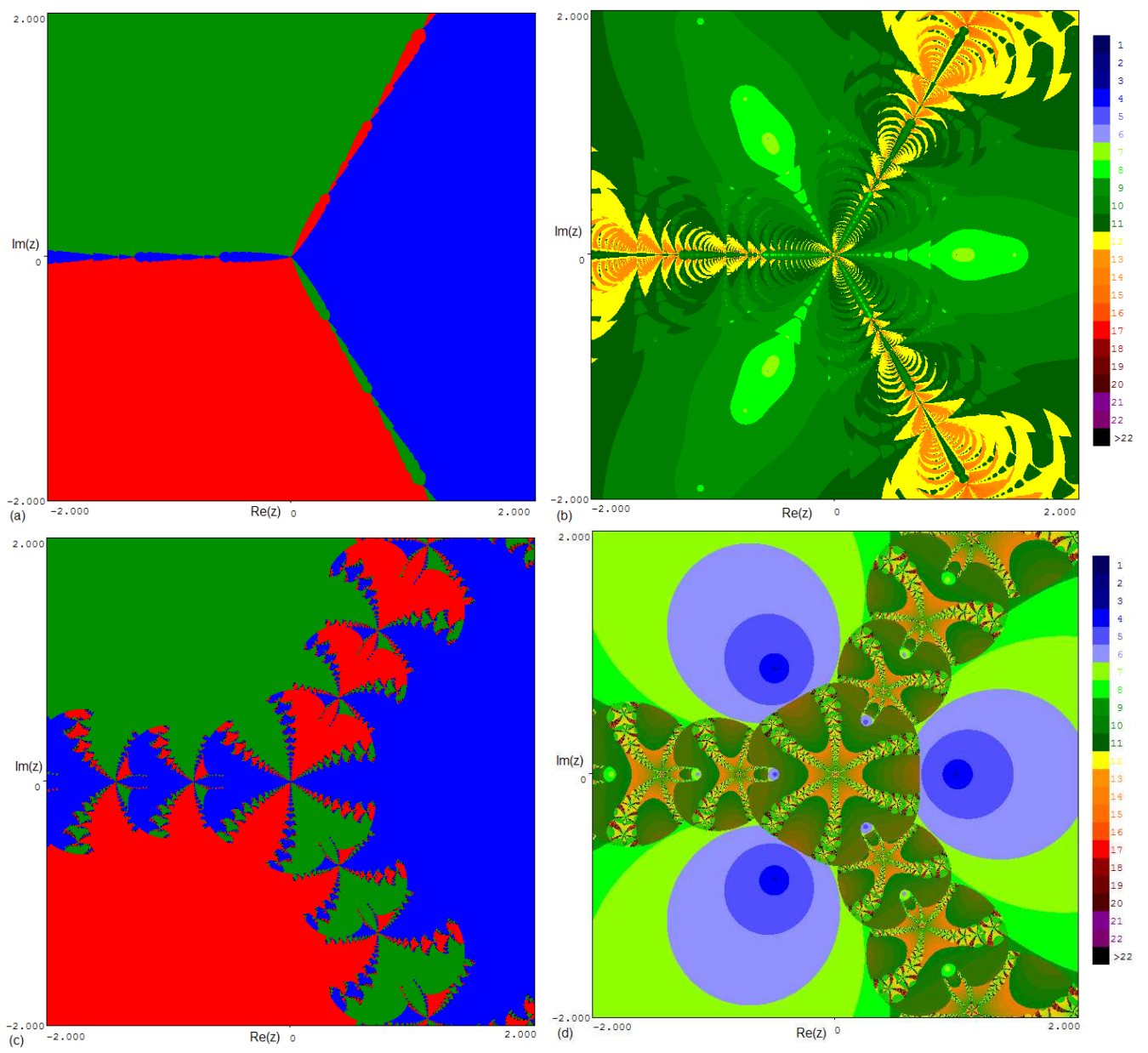

Figure 4. Bassin d'attraction des solutions de (3) utilisant l'algorithme (14) avec: (a)-(b) R.L. Wolfe. (c)-(d) R.L. Armijo. 
(6) s'écrit comme suit

$$
\left\{\begin{array}{l}
x_{+}=\frac{\lambda_{I}\left(x^{5}+x^{4} y+2 x^{2} y^{3}+2 x y\right)+\left(3-\lambda_{R}\right)\left(x^{5}+x y^{4}+2 x^{3} y^{2}\right)+\lambda_{R} x^{2}}{3\left(x^{2}+y^{2}\right)^{2}} \\
y_{+}=\frac{\left(3-\lambda_{R}\right)\left(y^{5}+x^{4} y+2 x^{2} y^{3}\right)-\lambda_{I}\left(x^{5}+x y^{4}+2 x^{3} y^{2}+y^{2}-x^{2}\right)-2 \lambda_{R} x y}{3\left(x^{2}+y^{2}\right)^{2}}
\end{array}\right.
$$

avec $\lambda=\lambda_{R}+i \cdot \lambda_{I} ;\left(\lambda_{R}, \lambda_{I}\right) \in \mathbb{R}^{2}$.

L'etude du $\lambda$-plan paramtrique (voir FIG. 5), montrent qu'il existe vraisemblablement une valeur optimale pour $\lambda$ dans le sens où la convergence est obtenue après une seule itération, cette valeur notée $\lambda^{*}$ peut être calculée en remplaçant dans l'équation (1) la quantité $z+\lambda \frac{1-z^{3}}{3 z^{2}}$, ce qui donne

$$
\left(z+\lambda \frac{1-z^{3}}{3 z^{2}}\right)^{3}-1=0
$$

En résolvant l'équation (9) par rapport à $\lambda$, on trouve

$$
\lambda^{*}=\frac{3 z^{2}}{z^{2}+z+1},
$$

plus précisément si on pose $\lambda^{*}=\lambda_{R}^{*}+i \cdot \lambda_{I}^{*}$, on obtient

$$
\left\{\begin{aligned}
\lambda_{R}^{*} & =\frac{3\left(x^{2}-y^{2}\right)\left(x^{2}-y^{2}+x+1\right)+6 x y(2 x y+y)}{\left(x^{2}-y^{2}+x+1\right)^{2}+(2 x y+y)^{2}} \\
\lambda_{I}^{*} & =\frac{6 x y\left(x^{2}-y^{2}+x+1\right)-3\left(x^{2}-y^{2}\right)(2 x y+y)}{\left(x^{2}-y^{2}+x+1\right)^{2}+(2 x y+y)^{2}}
\end{aligned}\right.
$$

Pour cette valeur, on a $\mathcal{J}\left(\mathcal{N}\left(\cdot, \lambda^{*}\right)\right)=\{\infty\} ; \operatorname{donc} \mathcal{F}\left(\mathcal{N}\left(\cdot, \lambda^{*}\right)\right)=\mathbb{C}$.

\section{Dimension fractale}

Le calcul de la dimension fractale est un moyen très efficace pour mesurer le degré d'irrégularité d'un ensemble. Il existe plusieurs types de dimensions fractales, parmi elles la dimension fractale de Hausdorff qui est la plus connue et la plus utilisée théoriquement [5]; Par contre son calcul numérique est très délicat. Dans ce travail, on utilise la méthode de " Comptage des boîtes " [7]. Pour ce faire l'ensemble $\mathcal{J}(\mathcal{N})$ est immergé dans une grille de taille $l$ (chaque cellule est carrée et a pour côté $l$ ). Si $N(l)$ désigne le nombre de cellules non vides contenant au moins un point de $\mathcal{J}(\mathcal{N})$, alors ; la dimension fractale de l'ensemble $\mathcal{J}(\mathcal{N})$ notée $d$ est donnée par

$$
d=\lim _{l \rightarrow 0} \frac{\ln N(l)}{-\ln (l)}
$$

Le moyen le plus utilisé pour obtenir $d$ est de calculer $\ln N(l)$ et $\ln (l)$ pour des valeurs de $l$ de plus en plus petites; on construit ainsi un nuage de points $\left(\ln N\left(l_{s}\right), \ln \left(l_{s}\right)\right), s=1,2, \ldots, p$ et on ajuste ce dernier par une droite aux moindres carrés. La pente de cette droite donne une estimation de la dimension $d$. Les résultats numériques montrant l'influence de la valeur numérique de $\lambda$ sur la dimension fractale sont résumés dans le tableau TAB. 1 . 

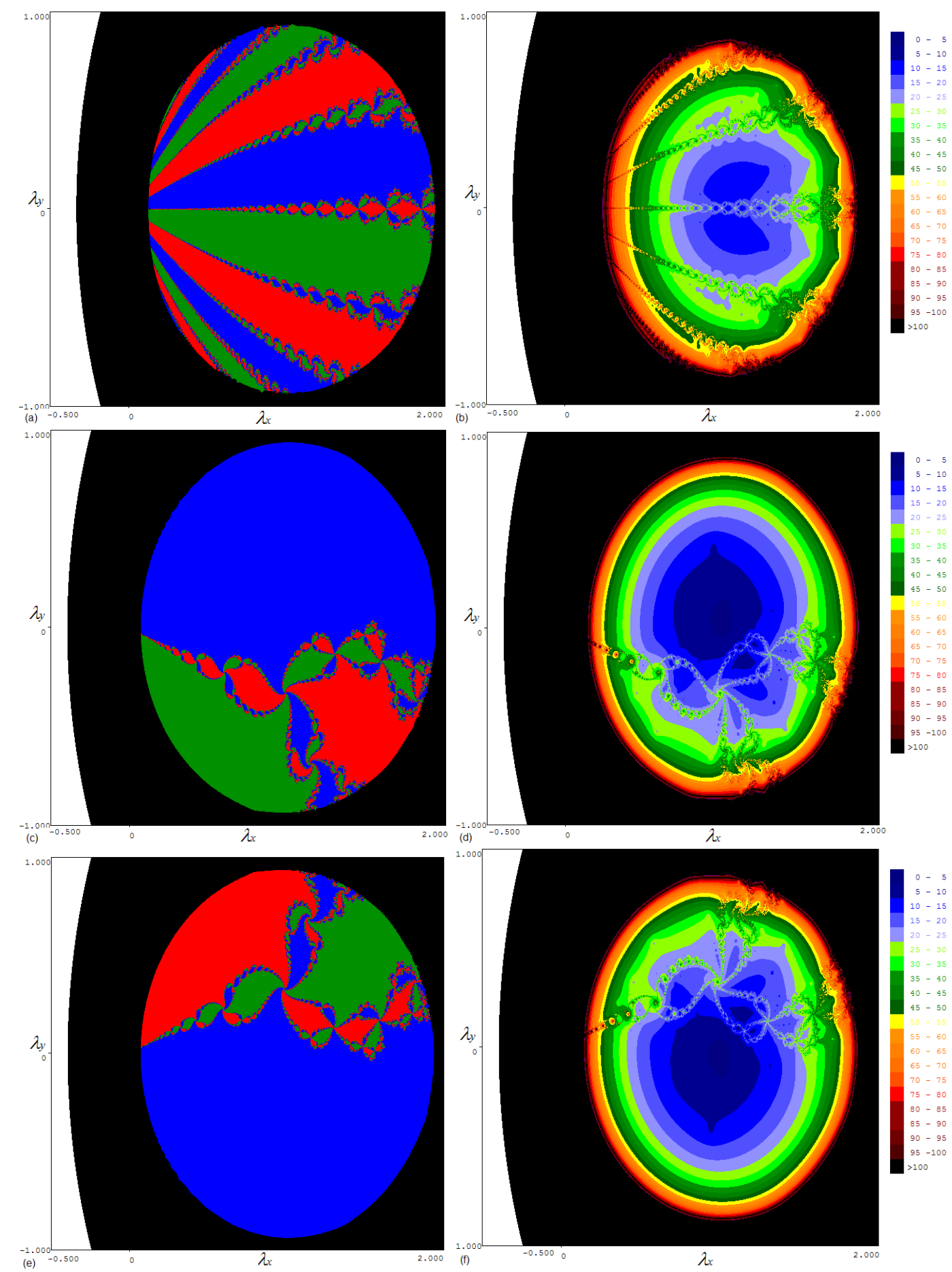

Figure 5. Le diagramme de bifurcation dans le plan $\left(\lambda_{R}, \lambda_{I}\right)$ pour l'algorithme de Newton modifié initié en: (a)-(b) $z_{0}=29.3443865317+i \cdot 50.82596840$. (c)-(d) $z_{0}=1.0+i \cdot 1.0$. (e)-(f) $z_{0}=1.0-i \cdot 1.0$. 
TABLE 1. La dimension fractale de l'ensemble de Julia $\mathcal{J}(\mathcal{N})$ en fonction de $\lambda$

\begin{tabular}{cc}
\hline \hline$\lambda$ & $d$ \\
\hline 0.00 & 1.00000 \\
0.10 & 1.23854 \\
0.20 & 1.19684 \\
0.40 & 1.22622 \\
0.60 & 1.30362 \\
0.80 & 1.31607 \\
1.00 & 1.35147 \\
1.20 & 1.36894 \\
1.40 & 1.41129 \\
1.60 & 1.43465 \\
1.80 & 1.57155 \\
$\lambda^{*}$ & 0.00000 \\
$\lambda_{A}$ & 1.43465 \\
$\lambda_{W}$ & 1.00983 \\
\hline \hline
\end{tabular}

\section{Conclusion}

Les calculs sur la dimension fractale montrent que le paramètre $\lambda>0$ est un facteur de complexité pour l'ensemble de Julia $\mathcal{J}(\mathcal{N})$ du fait que sa dimension fractale croit avec $\lambda$, alors que pour la valeur $\lambda=\lambda^{*}$ l'ensemble de Julia est détruit $\left(d=0\right.$ pour $\left.\lambda=\lambda^{*}\right)$. Les valeurs de la dimension fractale correspondant à $\lambda_{W}$ et $\lambda_{W}$ peuvent être interprétées par le fait que l'algorithme de Newton est plus performant avec la recherche linéaire de Wolfe par rapport à celle d'Armijo, ce qui se traduit par une meilleure régularité des frontières des bassins d'attractions $\left(d \approx 1.00983\right.$ qui est proche du 1.0 pour $\left.\lambda=\lambda_{W}\right)$.

\section{REFERENCES}

[1] M.S. Bazara, H.D. Sherali, C.M Shetty, Nonlinear programming. J.W\&S (1993)

[2] P. Blanchard, Complex analytic dynamics on the Riemann sphere, Bulltin of AMS, Vol. 11, Num 1, July 1984, 85-141.

[3] H.Brolin, Ivariant set under iteration of rational functions, Ark. Mat. 6(1965), 10-144.

[4] A. Cayley, Desiderata and suggestions. The Newton-Fourier Imaginary problem, Amer. J. Math. 2 (1879), no. $1,79$.

[5] M.R. Ferchichi and I. Djellit, Attracteurs étranges et dimensions fractales, Séminaire National sur les Fractales dans la Compression d'Images. Alger Mai 1998.

[6] G. Julia, Mémoire sur l'itération des fonctions rationnelles, J. Math. Pures et appl. 81(1918), 47-235.

[7] C. Lausberg, Calcul numérique de la dimension fractale d'un attracteur étrange, Thèse de doctorat, Institut National Polythechnique de Grenoble (I.N.P.G), (1992).

[8] J. Nocedal, Theory of algorithms for unconstrained optimization, acta Numerica, (1999), $199-242$.

[9] F. Von Haesler and H.-O. Peitgen, Newton's method and complex dynamical systems, Acta. Appl. Math. 13(1988), no. 1-2, $3-58$. 